\title{
V. Alliierte Prozesse gegen die Hauptkriegs- verbrecher: Tokio, Nürnberg und die Schulddebatte
}

Noch bevor der Kalte Krieg ab 1948 die amerikanische Besatzungspolitik in Japan - etwas später als jene in Deutschland - immer stärker zu prägen begann, hatten die USA eine Reihe von Schritten zur Bestrafung von Kriegsverbrechern und zur politischen Säuberung eingeleitet. Den Auftakt bildete das Internationale Militärtribunal für den Fernen Osten (auch Tokioter Prozeß genannt) vom Mai 1946 bis April 1948, dem bereits Erfahrungen mit dem - deutlich kürzeren - Nürnberger Kriegsverbrecherprozeß gegen führende Funktionsträger des Dritten Reiches (November 1945 bis Oktober 1946) zugrunde lagen. Wurde in Tokio den Hauptkriegsverbrechern (der sogenannten Kategorie A) der Prozeß gemacht, die der Verschwörung zum Angriffskrieg, der Verbrechen gegen den Frieden und die Menschlichkeit angeklagt waren, so beschäftigten die übrigen einzelnen Kriegsverbrechen (der Kategorie B und C), die meist vom einfachen Soldaten bis zum Befehlshaber von Kampfeinheiten in der Schlacht oder während der Besatzungszeit in den eroberten Länder verübt worden waren, den (amerikanischen) Militär-Gerichtshof in Yokohama, aber auch, was weniger bekannt ist, chinesische, britische, holländische, australische, philippinische und französische Gerichte. Dabei wurden bis 1951 insgesamt 4400 Japaner, Koreaner und Taiwanesen verurteilt und 920 Todesurteile vollstreckt ${ }^{1}$. Dic alliierten Gerichte in den Westzonen Deutschlands haben - zum Vergleich - 5000 Personen verurteilt, davon ca. 800 zum Tode. Etwa 500 Todesurteile wurden auch vollstreckt ${ }^{2}$. Hinzu kommen die Zahlen für den sowjetischen Machtbereich, die weit höher geschätzt werden ${ }^{3}$.

1 Cohen, Öffentliche Erinnerung, S. 53; Seraphim, Kriegsverbrecherprozesse in Asien, S. 82.

Steinbach, Nationalsozialistische Gewaltverbrechen, S. 29.

3 Gegen die an Polen ausgelieferten NS-Kriegsverbrecher wurde in 193 Fällen die Todesstrafe ausgesprochen. Zudem geht Richard J. Evans von einer Gesamtzahl von 300 Todesurteilen sowjetischer Militärtribunalc gegen Kriegsverbrecher in der SBZ aus. Hinter der offiziellen Zurückhaltung bei der gesetzlichen Anwendung der Todesstrafe verbarg sich jedoch eine ganz andere Situation in den von der sowjetischen politischen Polizei eingerichteten Speziallagern für wirkliche oder angebliche Nazis. Dort wurden auf Befehl der Lagerleitung ohne jedes Gerichtsurteil mindestens 876 Häftlinge erschossen. Andere Quellen sprechen von 78000 bis 96000 Toten. Evans, Rituale der Vergeltung, S. $959 \mathrm{f}$. Vgl. auch Meyer-Seitz, Die Verfolgung von NS-Straftaten. Zur Schwierigkeit, genaue Zahlen zu ermitteln, siehe Wentker, Die juristische Ahndung von NS-Verbrechen, S. 62. 
Beim Tokioter Prozeß waren zu Beginn 28 Personen angeklagt, darunter vier ehemalige Premierminister, der prominenteste unter ihnen Tôjô Hideki, sowie elf Minister, zwei Ex-Botschafter und acht hochrangige Generäle; im Verlauf des Verfahrens starben allerdings zwei der mutmaßlichen Hauptkriegsverbrecher, während ein weiterer wegen einer eingetretenen Geisteskrankheit aus dem Verfahren genommen werden mußte. Die Todesstrafe wurde schließlich gegen sieben der Angeklagten verhängt und am 23. Dezember 1948 durch den Strang vollzogen. 18 der zivilen und militärischen Führer hatten Gefängnisstrafen zu verbüßen, 16 davon potentiell lebenslänglich; allerdings starben nur sechs Mitglieder dieser Gruppe im Gefängnis, die anderen zwölf kamen nach Verbüßung lediglich eines Teils ihrer Strafen auf freien Fuß ${ }^{4}$.

Von der Größe und der Symbolträchtigkeit des Ortes her war der Tokioter Prozeß, der im Auditorium Maximum der früheren Kaiserlichen Militärakademie stattfand, wo etliche der jetzt Angeklagten dereinst Festreden gehalten hatten, mit dem Hauptkriegsverbrecherprozeß in Nürnberg, der Stadt der NSDAP-Reichsparteitage, in gewisser Weise vergleichbar ${ }^{5}$. Dies galt auch für Bedenken, die wegen des Rückwirkungsverbots des Strafrechts, aber auch aufgrund der prinzipiellen Unteilbarkeit des Völkerrechts in Japan wie in Deutschland dagegen aufkamen, daß hier nur über die Verbrechen der Besiegten, nicht aber über Katyn oder Nagasaki ein Urteil gefällt wurde 6 . Dem entsprach auch die Auswahl der Richter und Staatsanwälte, die beim Tokioter Militärtribunal hauptsächlich von den vier Siegermächten mit asiatischem Kolonialbesitz gestellt wurden, wohingegen asiatische Juristen aus Burma, Indonesien, Malaysia, Singapur und Vietnam, in denen japanische Truppen zahlreiche Verbrechen begangen hatten, kaum und neutrale Staaten gar nicht vertreten waren7. Als Strafverteidiger fungierten sowohl amerikanische wie einheimische Rechtsanwälte, wobei letztere aufgrund der Orientierung des japanischen Strafprozeßrechts am deutschen Vorbild ähnliche Schwierigkeiten mit dem nach anglo-amerikanischen Muster durchgeführten Verfahren hatten wie ihre Kollegen in Nürnberg. Darüber hinaus erwiesen sich beim Tokioter Prozeß nicht nur die Sprach- und Übersetzungsprobleme als ungleich gravierender, auch beim Niveau des juristischen Personals gab es beträchtliche Unterschiede. Robert

4 Vgl. Minear, Victor's justice, S. 172.

5 In Nürnberg erfolgten zwölf Todesurteile, drei lebenslängliche und vier befristete Freiheitsstrafen sowie drei Freisprüche. Einen guten Überblick über die Nürnberger Prozesse bietet neben Wieland, Der Jahrhundertprozeß, jetzt auch Kastner, Von den Siegern. Zum Vergleich beider Prozesse vgl. auch Dower, Embracing Defeat, S. 454-461, sowie Röling/Cassese, The Tokyo Trial and Beyond, S. 2f., $89 \mathrm{ff}$.

6 Vgl. Awaya, The Tokyo Trials and the BC Class Trials, S. 45, sowie Osten, Die japanische Strafrechtswissenschaft und der Tokioter Prozeß, S. $100 \mathrm{f}$.

7 Die beteiligten Juristen kamen aus elf Ländern: USA, Großbritannien, China, UdSSR, Niederlande, Frankreich, Australien, Neuseeland, Kanada, Indien, Philippinen. 
Jackson, US-Chefankläger in Nürnberg, galt als brillanter und sendungsbewußter Jurist, im Vergleich dazu war sein Tokioter Pendant Joseph B. Keenan, der den Job, wie es hieß, vor allem zur Profilierung für einen Sitz im US-Senat nutzen wollte, medioker, ja der niederländische Richter hielt ihn ebenso wie den australischen Gerichtspräsidenten für „eindeutig unqualifiziert" 8 .

Während in Nürnberg der Hauptpunkt der Anklage gegen 24 Spitzenfunktionäre der NS-Diktatur auf „Verschwörung zur Planung, Vorbereitung und Durchführung eines Angriffskrieges" lautete, ging es in Tokio lediglich um die „Verschwörung zum Angriffskrieg“. Der Straftatbestand des Verbrechens gegen den Frieden mit seiner Individualhaftung für den Angriffskrieg stand im Mittelpunkt. Anders als in Nürnberg wurde in Tokio aber kein einziger Angeklagter wegen Verbrechen gegen die Menschlichkeit verurteilt ${ }^{9}$. Den Nürnberger Richtern fiel angesichts der offensichtlichen Planmäßigkeit der nationalsozialistischen Aggression das Verdikt von einem ungerechten Krieg nicht schwer, wohingegen die Unterscheidung zwischen einem bellum iustum bzw. iniustum auf dem Tokioter Tribunal zu erheblichen Kontroversen führte - bis hin schließlich zum Sondervotum des auf Freispruch für alle Angeklagten plädierenden Richters aus Indien, wo man die 1947 erreichte Befreiung von britischer Kolonialherrschaft nicht zuletzt auf den von Japan begonnenen Krieg im Fernen Osten zurückführte ${ }^{10}$.

Zu den Spezifika des Tokioter Tribunals zählte nicht nur das starke Interesse der japanischen Rechtsanwälte und ihrer Mandanten daran, die Vereinbarkeit der japanischen Kriegführung mit dem Völkerrecht sowie die Unvermeidbarkeit des Krieges für die japanische Seite nachzuweisen, sondern vor allem auch ihre Neigung, jede Verantwortung des Tennô in Abrede zu stellen. Wie nur wenige der Nürnberger Angeklagten zeigten sich die japanischen Politiker und Militärs vor den Tokioter Richtern auch bereit, strafrechtliche Verantwortung auf sich zu nehmen, um der japanischen Verteidigungsstrategie zu Gunsten des Tennô zum Erfolg zu verhelfen. So bezichtigten sie sich vor dem Tribunal selbst, die Kriege allein geplant zu haben, während der stets auf Frieden bedachte Kaiser bei den entsprechenden Sitzungen nur aus protokollarischen Gründen als Ehrengast präsent gewesen sei. Mit Verweis auf politische Handlungen der alliierten Mächte, die ebenfalls den Krieg begünstigt hätten, meinten die Angeklagten aber lediglich gegenüber dem Kaiser schuldig geworden zu sein: „Eine andere Schuld erkannten sie nicht an. Ihre Absicht war, sich aus Treue für ihren Kaiser hin-

8 So der niedcrländische Richter am Tokioter Militärtribunal:Röling, Introduction, S. 16 f.

9 Osten, Dic japanische Strafrechtswissenschaft, S. 94 f.; bis auf zwei Angeklagte, die „nur“ wegen Kriegsverbrechen verurteilt wurden, befanden die Richter alle für schuldig, sich zur Führung cines Angriffskriegs verschworen zu haben.

10 Vgl. Martin, Japan und der Krieg, S. 193, 195 f. 
zuopfern."11 Vorschläge von amerikanischer Seite, sich um den Nachweis individueller Unschuld ihrer Mandanten zu bemühen, lehnten die japanischen Verteidiger demgegenüber einstimmig ab.

Daß Hirohito der Anklage entging, war sicher das größte und entscheidende Manko des Tokioter Tribunals. Denn ob die Aufnahme seines engsten Beraters, ja politischen Alter egos Graf Kido Kôichi in den Kreis der Angeklagten tatsächlich auf eine subtile, aber vom japanischen Volk verstandene Art und Weise für die indirekte Präsenz des Tennô im Prozeß sorgte, ist fraglich ${ }^{12}$. Weitere schwerwiegende Fehler kamen hinzu. So konnte der japanischen Öffentlichkeit schlechterdings nicht entgehen, wie sehr sich das Militärtribunal trotz seines hohen moralischen Anspruchs auf japanische Verbrechen gegen westliche Opfer konzentrierte, obwohl diese weniger als ein Zehntel der Gesamtopfer der japanischen Aggression ausmachten ${ }^{13}$. Zudem wurden weder die wichtigsten Führer der kempeitai d.h. der japanischen Version von Geheimer Staatspolizei - noch die der ultranationalistischen Geheimgesellschaften und der zaibatsu, also der großen, in die Kriegsrüstung verstrickten Wirtschaftskonzerne, in Tokio zur Anklage gebracht ${ }^{14}$. Auch das "Comfort-Woman-System" wurde nicht in die Anklagepunkte des Prozesses aufgenommen, nachdem die japanische Militärbürokratie die entsprechenden belastenden Dokumente noch vor Eintreffen der amerikanischen Besatzungsarmee fast vollständig vernichtet hatte ${ }^{15}$.

Eine besondere Beeinträchtigung aber erfuhr das Prestige des Prozesses zweifelsohne durch das Bekanntwerden des japanischen Laboratoriums für bakteriologische Waffen in der Mandschurei, in dem Tausende Gefangene ihr Leben verloren hatten ${ }^{16}$. Da zentral ausgegebene Befehle, wie sie der Nürnberger Anklage zur Verfügung standen, in Tokio selten zu finden waren, kam diesem Fall systematisch organisierter japanischer Kriegskriminalität potentiell eine Schlüsselrolle zu. Doch die US-Militärbehörden wollten sich um jeden Preis der Ergebnisse der mandschurischen Forschung bemächtigen und sie nicht in die Hände der Sowjets fallen lassen. Im Gegenzug für die Lieferung ihres Wissens über die Experimente wurde den in die Verbrechen involvierten Japanern Immunität versprochen ${ }^{17}$. Die Sowjets revanchierten sich, indem sie Ende 1949 in Chabarowsk einen Prozeß gegen zwölf ehemalige Angehörige der japanischen Armee „wegen Vorbereitung

11 Miyazawa, Rechtsprobleme, S. 31.

12 Brackman, The other Nuremberg, S. 86.

13 Halliday, Japan unter amerikanischer Besatzung, S. 121.

$14 \mathrm{Vgl}$. Brackmann, The other Nuremberg, S. 85.

15 In der Folge wurde die bloße Existenz der 50000 bis 200000 Frauen bis Anfang der 1990er Jahre von der japanischen Regierung geleugnet. Vgl. Osten, Die japanische Strafrechtswissenschaft, S. 95.

16 Vgl. hierzu Harris, Factories of Death.

17 Vgl. Röling, Introduction, S. 18 f. 
und Anwendung der Bakterienwaffe" durchführten, in dessen Verlauf nicht nur die Rolle des Kriegsministers, sondern auch die des Kaisers ins Visier geriet $^{18}$ - zumal als im Dezember 1950 auch Mao forderte, Hirohito und die leitenden Forscher wegen bakteriologischer Kriegsführung vor Gericht zu bringen ${ }^{19}$.

Das japanische Volk, das mit Extrablättern in den amerikanisch kontrollierten und entsprechend orientierten Zeitungen sowie in dem von der Besatzungsmacht produzierten Rundfunkprogramm („Die Büchse der Wahrheit") über die entscheidenden Stationen des Tokioter Prozesses unterrichtet wurde, akzeptierte ihn japanischer Tradition entsprechend wie eine unvermeidliche Naturkatastrophe 20 . Es zeigte in seiner Mehrheit jedenfalls keine heftige Empörung gegen die ausgesprochenen Todesurteile - sieht man einmal von dem auf Unverständnis stoßenden uneinheitlichen Richterspruch gegen Hirota Kôki ab, den starken Mann der japanischen Politik in den 1930er Jahren und Außenminister zur Zeit des Massakers von Nanking, für den eine Petition mit 300000 Unterschriften vergeblich um Gnade bat. Manche Japaner hielten das ganze Gerichtsverfahren von vornherein für überflüssig, weil die Angeklagten am Tag der Kapitulation besser hätten seppuku (harakiri) begehen sollen ${ }^{21}$. Wäre der Tokioter Prozeß in japanischer Regie durchgeführt worden, hätte aber wohl nicht die Frage der Verantwortung der Führung für den Kriegsbeginn im Mittelpunkt gestanden, sondern deren Schuld an der Niederlage und der danach ausgebrochenen Hungersnot, die in besonders betroffenen Landesteilen damals das Hauptproblem einer ums nackte Überleben kämpfenden Bevölkerung war ${ }^{22}$. Immerhin meinten etliche Zeitungen in der Provinz nach dem Urteilsspruch, der Kaiser könne seine Verantwortung für Kriegsverbrechen nun nicht länger verleugnen und solle abdanken, um das japanische Volk durch diesen Schritt in Schutz zu nehmen ${ }^{23}$. Vor allem Intellektuelle wie der junge Prozeßbeobachter Miyazawa Kôichi gelangten zu der Einsicht, „daß die Angeklagten einer zwingenden und gerechten Bestrafung zugeführt wurden“24.

Die Rezeption des Prozesses in der Öffentlichkeit war zum einen durch die Politik MacArthurs beeinflußt, der prinzipielle Aversionen dagegen hatte, die politischen Führer einer besiegten Nation im kriminellen Sinne

$18 \mathrm{Vgl}$. Prozeßmaterialien in der Strafsache gegen ehemalige Angehörige der japanischen Armee wegen Vorbereitung und Anwendung der Bakterienwaffe.

19 Seraphim, Kriegsverbrecherprozesse in Asien, S. 83.

20 Tsurumi, A Cultural History of Postwar Japan, S. 19 f.; Ishida, Das Massaker von Nanking, S. 236.

21 So hatte auch Tôjô Hidekis Reputation erheblich gelitten, weil er in der Stunde der Niederlage nicht rituell seppuku begangen, sondern versucht hatte, sich mit der Pistole das Leben zu nehmen. Scharlau, Der General und der Kaiser, S. 89.

22 So Tsurumi, What the War Trials left to the Japanese People, S. 137.

23 Vgl. Brackman, The other Nuremberg, S. 395.

${ }^{24}$ Miyazawa, Rechtsprobleme, S. 34. 
schuldig zu sprechen. Da die Alliierten und die amerikanische Öffentlichkeit aber, wie er klagte, unbedingt Blut sehen wollten, konnte sich der General mit seiner Position in diesem Fall einmal nicht durchsetzen. Überzeugt, es beim Militärtribunal „mit der schlimmsten Hypokrasie der bekannten Geschichte" zu tun zu haben, setzte MacArthur zumindest alles daran, die Gefühle der Japaner weitestgehend zu schonen und ließ - entgegen einer Weisung aus Washington - Photographen bei der Hinrichtung nicht $\mathrm{zu}^{25}$. Zur Rezeptionsgeschichte des Prozesses gehört es ferner, daß das Volk sich zum damaligen Zeitpunkt noch kein Gesamtbild des Krieges machen konnte. Berichte über die Atombombenabwürfe wurden von SCAP per Zensur unterbunden ${ }^{26}$. Hätten die Japaner damals schon die Zweifel an der militärischen Notwendigkeit des A-Waffen-Einsatzes in Hiroshima und vor allem Nagasaki gekannt, wäre die Reaktion auf den Prozeß sicher anders, kritischer ausgefallen.

Als sie die Tatsachen dann mit Verspätung kurz nach Beendigung der amerikanischen Besatzung 1952 kennenlernten, hatte dies eine um so problematischere Wirkung auf ihr Kriegsschuldbewußtsein. Denn die daraus resultierende, wenn auch erst allmählich sich voll ausprägende Opferperspektive ${ }^{27}$ ließ den Tokioter Prozeß in der Retrospektive mehr und mehr als einen Akt des Machtmißbrauchs von Siegermächten erscheinen und machte den von den USA intendierten moralischen Einfluß, in welchem Umfang auch immer er vom Kriegsverbrechertribunal ausgegangen war, wieder weitgehend zunichte. So konnten Bücher wie das über den 1948 als Kriegsverbrecher hingerichteten Hirota ${ }^{28}$ später zu Bestsellern avancieren ${ }^{29}$. Bei einer Meinungsumfrage zum Tokioter Prozeß äußerten 1955 rund $60 \%$ die Ansicht, daß das Gericht der Alliierten nicht zu billigen gewesen sei ${ }^{30}$, ganz anders als noch zu Beginn des Verfahrens, als die meisten Japaner einverstanden damit gewesen waren, daß ihrer politischen Führung der Prozeß gemacht wurde 31 .

Die Parallelen dieser Entwicklung zur Aufnahme des Nürnberger Prozesses in Deutschland sind mit den Händen zu greifen. Wurde die Anklage gegen die Hauptkriegsverbrecher hier anfangs von einer klaren Mehrheit als fair und notwendig angesehen und die Strafen als angemessen, so dachte vier

25 Scharlau, Der General und der Kaiser, S. 84, 91 (Zitat).

$26 \mathrm{Vgl}$. Braw, The Atomic Bomb Suppressed.

27 Vgl. grundlegend den vom Committee for the Compilation of Materials on Damage Caused by the Atomic Bombs herausgegebenen Band: Hiroshima and Nagasaki, sowie Dower, The Bombed: Hiroshima and Nagasaki in Japanese Memory.

28 Shiroyama, War Criminal. The life and Death of Hirota Kôki (so der Titel der 1977 erschienenen englischen Übersetzung).

29 Tsurumi, What the War Trials left to the Japanese People, S. 139.

30 Ida, Strafverfolgung und Schuldauffassung der Japaner; S. 110.

31 Vgl. hierzu die Einschätzung des Nippon-Times-Herausgebers Kawai Kazuo; zit. bei Benz, Amerikanische Besatzungsherrschaft in Japan, S. 275. 
Jahre später nur mehr ein Drittel so ${ }^{32}$. In beiden Ländern war dies nicht zuletzt darauf zurückzuführen, daß man sich von der Anklage gegen die Hauptkriegsverbrecher zunächst nicht persönlich betroffen fühlen mußte, sondern vielmehr in der Vorstellung leben konnte, von einigen offensichtlich verbrecherischen Führern arglos getäuscht und mißbraucht worden zu sein. Doch wer glaubte, sich dergestalt der eigenen Mitverantwortung dauerhaft entziehen zu können, wurde schon bald eines besseren belehrt, als die politische Säuberung durch die Besatzungsbehörden Hunderttausende auch weniger stark Belastete und selbst Mitläufer erfaßte. Zudem boten die prozeduralen Defizite im Rahmen von Entnazifizierung bzw. Entmilitarisierung reichlich Anlaß, das ganze Verfahren in Zweifel zu ziehen.

Die Parallelen zwischen Nürnberger und Tokioter Tribunal vermögen aber einen entscheidenden Unterschied nicht zu verwischen: Während der Jahrhundertprozeß von Nürnberg ${ }^{33}$ die deutsche Schuld klar und deutlich dokumentiert und ins kollektive Gedächtnis der Nation wie der Menschheit eingemeißelt hat, konnte der Vergebungsakt MacArthurs für den Kaiser in Japan wie in der Weltöffentlichkeit auch als pauschale Entlastung des japanischen Volkes mißverstanden werden ${ }^{34}$. Wenn der Tennô, „obwohl er die formelle Verantwortung für alles, was geschehen war, getragen hatte, dafür nicht zur Rechenschaft gezogen wurde, konnte auch alle anderen keine Schuld treffen" 35 . Vor diesem Hintergrund ist Burumas Vorwurf, mit der Verschonung des Tennô habe sich MacArthur „der Geschichtsfälschung schuldig“ 36 gemacht, kaum ganz von der Hand zu weisen. Sogar der Film „Die japanische Tragödie“ (1946), in dem der linksgerichtete Regisseur Kamei Fumio die Rolle des Kaisers während des Krieges scharf kritisierte, ließ er als subversiv verbieten, nachdem Ministerpräsident Yoshida Shigeru bei der US-Besatzungsbehörde Protest eingelegt hatte ${ }^{37}$.

Hinzu kam, daß sich auch in der Folgezeit das amerikanische Interesse an der Publizität des Tokioter Prozesses in engen Grenzen hielt. So erachtete es Washington - anders als nach Abschluß des Nürnberger Verfahrens - nicht für tunlich, Tokioter Prozeßakten „in einer großangelegten Dokumentation den Japanern als Sündenregister vorzuhalten " 38 . Die in wenigen amerikanischen Bibliotheken vorhandenen Prozeßmaterialien wurden erst 1957 durch einen Indexband erschlossen und erst 1977, fast 30 Jahre nach Urteilsverkündung, publizierten zwei niederländische Juristen den vollen Wortlaut

32 Reichel, Vergangenheitsbewältigung, S. 69.

33 Wieland, Der Jahrhundertprozeß von Nürnberg.

34 Vgl. hierzu auch die Auffassung von Sato Tadao, zit. nach Awaya, The Tokyo Trials, S. 45.

35 Buruma, Erbschaft, S. 225.

36 Ebd., S. 222.

37 Ebd., S. 225.

38 Martin, Japan und der Krieg, S. 193. 
des Gesamturteils ${ }^{39}$. Daß der Nürnberger Prozeß ungleich besser erforscht wurde als sein Tokioter Pendant, kann mithin kaum verwundern ${ }^{40}$.

Die erwähnten Parallelen in der nationalen Rezeption des Nürnberger und Tokioter Prozesses dürfen schließlich nicht darüber hinwegtäuschen, daß sich die Diskussion über die Schuld an Krieg und Verbrechen in Deutschland und Japan von Anfang an sehr unterschiedlich entwickelte. Dazu beigetragen hatten sicherlich die großen Meinungsverschiedenheiten zwischen den - nicht nur vier, wie in Nürnberg, sondern elf - Tokioter Richtern, die es für die Japaner „sozusagen legitim“ werden ließen, „die Urteile ihrerseits politisch auszuwerten " 41 . Obendrein ist $\mathrm{zu}$ bedenken, daß der Tennô bereits in seinem „Kaiserlichen Edikt zum Kriegsende“ am 15. August 1945 die japanischen Opfer, d.h. die Vernichtung „vieler unschuldiger Menschenleben“ durch den „neuen grausamen Bombentyp“, herausgestellt hatte. Der Verteidigungskrieg, so bekräftigte der Kaiser, sei im Interesse der Existenz der Nation geführt worden. Von Schuld sprach er nicht, sondern bedauerte lediglich die asiatischen Verbündeten Japans, die mit davon betroffen seien, daß sich der Krieg „nicht zu Japans Gunsten entwickelt" habe 42 .

Zum ersten Mal brachte Higashikuni Naruhiko, der in der Übergangszeit vom Krieg zur Besatzung als Premierminister amtierte, in einem Interview am 28. August die Frage der Verantwortung für den Krieg öffentlich zur Sprache. Er appellierte an die alte Militärführung, die Ministerialbürokratie und das ganze Volk, über die Geschehnisse der jüngsten Vergangenheit nachzudenken und diese zu bereuen ${ }^{43}$. Nach shintôistischem Verständnis wird durch göttliche Gnade gereinigt, wer seine Vergangenheit bereut, wobei die Vergebung allerdings nicht wie im christlichen Denken auch innere Wandlung voraussetzt ${ }^{44}$. Insofern blieb der Begriff der „Gemeinsamen Reue von 100 Millionen“, zu der auch die Medien das Volk aufforderten, ein wenig nebulös und führte eher zur Verwischung von Verantwortlichkeiten $^{45}$. In Leserbriefen an die Zeitungen wiesen vor allem auch einfache Arbeiter auf ihre geringen politischen Einflußmöglichkeiten unter dem alten System hin, wobei sie unterstützt wurden von Intellektuellen, die Militär, Hofadel und hohe Bürokratie für den Angriffskrieg verantwortlich mach-

39 Röling/Rüter (Hg.), The Tokyo Judgement.

40 So enthält etwa John R. Lewis' einschlägige Bibliographie der Kriegsverbrecherprozesse 1290 Titel zu Nürnberg und nur 231 zu Tokio. Lewis, Uncertain Judgement.

41 Seraphim, Kriegsverbrecherprozesse in Asien, S. 84.

42 Die in altertümlichem Hofjapanisch abgegebene Erklärung wurde zwar nur von den wenigsten Zuhörern verstanden, aber von einem Rundfunksprecher in die Alltagssprache übertragen. Eine deutsche Übersetzung der Ansprache hat Gerhard Krebs in: Periplus 5 (1995), S. $50 \mathrm{f}$, vorgelegt.

43 Iritani, Group Psychology of the Japanese, S. $215 \mathrm{ff}$.

44 Ebd., S. 295.

45 Vgl. Miyazawa, Rechtsprobleme, S. 35, sowie Coulmas, Das Land der ritucllen Harmonie, S. 65. 
ten ${ }^{46}$. Dem hielt Premierminister Shidehara Kijûro im Dezember 1945 vor dem Oberhaus entgegen, daß die für den Krieg Verantwortlichen keine schlechten Absichten gehabt hätten ${ }^{47}$. Bald wurde immer deutlicher, daß eine übergroße Mehrheit des Volkes - im Gegensatz zu den ins Abseits geratenden marxistischen Kräften - enorme Schwierigkeiten hatte, zu Lebzeiten des verehrten Tennô überhaupt noch von Kriegsschuld und Kriegsverbrechen zu reden.

Im Westen Deutschlands ist die Frage der Schuld aufs Ganze gesehen gründlicher und teils auch selbstkritischer diskutiert worden. Zwar kam die amerikanische Information Control Division zu dem Schluß, daß die zahlreichen Kinobesucher, die sich etwa um die Jahreswende 1945/46 den KZFilm „Die Todesmühlen“ angesehen hatten, in ihrer Mehrheit keine Mitverantwortung für die Greuel anerkannten, doch im britischen Foreign Office war man zuversichtlich, daß die Langzeitwirkung derartiger Filme davon abhänge, wie jene Gruppe von Deutschen reagierte, die in Zukunft „die öffentliche Meinung prägen werde“48. Tatsächlich bewegte die Frage, „Wer war schuld an Hitler?" während der ersten Nachkriegsjahre zahllose Autoren in den neuen kulturell-politisch-literarischen Zeitschriften, die oft noch $1945 \mathrm{zu}$ Hunderten aus dem Boden gesprossen waren ${ }^{49}$. Besondere Beachtung fand eine Vorlesungsreihe des Heidelberger Philosophen Karl Jaspers im Wintersemester 1945/46, die nicht zuletzt auf die Frage einging, „in welchem Sinn sich jeder von uns mitverantwortlich fühlen muß“. Jaspers kam im Blick auf die „viele[n] von uns, die in ihrem innersten Wesen Gegner all dieses Bösen waren und durch keine Tat ... in sich eine moralische Mitschuld anzuerkennen brauchen", zu dem Schluß: Kollektivschuld gebe es „Zwar notwendig als politische Haftung der Staatsangehörigen, nicht aber darum im gleichen Sinne als moralische und metaphysische und nicht als kriminelle Schuld" ${ }^{50}$. Im Zusammenhang mit der von ihm betonten kollektiven politischen Haftung sprach Jaspers von der Notwendigkeit tiefgreifender innerer Reinigung und Buße. Im September 1945 bekannte sich auch der Rat der Evangelischen Kirche in Deutschland zur "Solidarität der Schuld“, in der „wir uns mit unserem Volk ... wissen“ und klagte sich „mit großem Schmerz“ an: „Durch uns ist unendliches Leid über viele Völker und Länder gebracht worden" 51 .

46 Schwentker, Täter oder Opfcr?, S. 151.

47 Iritani, Group Psychology of the Japanese, S. 226.

48 Chamberlin, Todesmühlen, S. $435 \mathrm{f}$.

49 Vgl. Eberan, Wer war schuld an Hitler?, sowie Wolgast, Die Wahrnehmung des Dritten Reiches, der Debatten im Bereich von Parteien, Kirchen und Universitäten nachzeichnet.

50 Jaspers, Die Schuldfrage, S. 56.

51 Weber, Auf dem Wege zur Republik, S. $112 \mathrm{f}$. 
Neben erschütternden Häftlingsberichten wie Wolfgang Langhoffs „Die Moorsoldaten" 52 oder Walther Pollers „Arztschreiber in Buchenwald“ erschien schon ein Jahr nach Kriegsende die bis heute lesenswerte Analyse Eugen Kogons über den „SS-Staat“. Zeitgleich entstanden Dramen wie Günther Weisenborns „Die Illegalen“, das den kommunistischen Widerstand gegen Hitler noch 1945 zum Thema machte und an über 350 Bühnen aufgeführt wurde. Thomas Manns metaphysische Deutung des Phänomens Nationalsozialismus in seinem 1947 erschienenen Roman „Doktor Faustus" war also nur das prominenteste Beispiel für eine frühe und intensive Auseinandersetzung mit dem Dritten Reich, die von den ehemaligen Verfolgten besonders engagiert vorangetrieben und darüber hinaus vor allem von jenen Schichten des Volkes geführt wurde, die auch sonst Bücher und Kulturzeitschriften lasen oder ins Theater gingen. Daß die Schulddiskussion vor allem im intellektuellen Milieu geführt wurde, während die breite Masse des Volkes persönliche Verantwortung für das Geschehene eher zurückwies, war in Japan tendenziell ähnlich. Allerdings kreiste die öffentliche Debatte hier eher um die Schuld an der Niederlage als um die Schuld an den Verbrechen, die während des Krieges verübt worden waren.

Der bedeutsamste Unterschied zwischen deutscher und japanischer Entwicklung betraf die Haltung der politischen Führungsschicht. Von der Spitze des neuen bundesdeutschen Staates her klangen die Bewertungen der Vergangenheit jedenfalls deutlich weniger exkulpierend als bei den ersten japanischen Premierministern, auch wenn es zum Signum dieser frühen „Reden nach Hitler“ zählte, „grundsätzlich Schuld zu bekennen, ohne sie individuell zuzurechnen" ${ }^{53}$. Die Zuschauerrolle der deutschen Bevölkerung „im Hinblick auf eine mögliche Verfehlung durch Unterlassen“ wurde also in der Regel ebenso wenig problematisiert wie Ausmaß und Intensität der Schuldverstrickung ${ }^{54}$. Statt dessen gab es in allen Parteien Tendenzen zu einem schuldentlastenden Opferbewußtsein, so als ob „im Grunde das ganze deutsche Volk“ zu den Opfern des Nationalsozialismus und „seiner dämonischen Zwecke“ zu zählen sei ${ }^{55}$. Freilich ist damit nur die eine Seite des ambivalenten westdeutschen Schulddiskurses umschrieben, es gab auch eine andere.

Bundespräsident Theodor Heuss wies etwa im Dezember 1949 in einer Rede vor der Gesellschaft für Christlich-Jüdische Zusammenarbeit die hinter dem Wort „Kollektivschuld“ steckende „simple Vereinfachung“ zurück,

52 Das bereits 1935 in Zürich erschienene Buch wurde 1946 erstmals legal in Deutschland von einem Münchner Verlag herausgebracht.

53 Baumgärtner, Reden nach Hitler, S. 342. Zu den Ähnlichkeiten der Position von Heuss mit der Konrad Adenauers und Kurt Schumachers vgl. ebd., S. 119-132.

54 Ebd.

55 So äußerten sich in den ersten Nachkriegsjahren der Ministerpräsident Schleswig-Holsteins, Theodor Steltzer (CDU), bzw. der Hamburger Bürgermeister Max Brauer (SPD). Reichel, Vergangenheitsbewältigung, S. 68. 
betonte aber gleichzeitig, daß aus der Hitler-Zeit „eine Kollektivscham ... gewachsen und geblieben“ sei ${ }^{56}$. Damit trug Heuss einerseits "der damaligen mentalen Situation in Deutschland Rechnung", unternahm aber auch den Versuch, das Postulat der Erinnerung an die NS-Diktatur im „normative[n] kollektive[n] Gedächtnis“ der Bevölkerung zu verankern ${ }^{57}$. Im November 1952 bei der Einweihung der Gedenkstätte Bergen-Belsen sprach der Bundespräsident von der "volle[n] Grausamkeit der Verbrechen, die hier von Deutschen begangen wurden“ und zählte „auch Auschwitz“ bereits zu einem „Katalog des Schreckens und der Scham“58. Dem untergegangenen Regime „keine schlechten Absichten“ zuzubilligen, wie dies Shidehara in bezug auf die ultranationalistische Regierung getan und wie dies auch der Tennô zu erkennen gegeben hatte, war eine Aussage, deren Verbreitung sich in Westdeutschland eher auf die Stammtische konzentriert haben dürfte. Denn auch hier hielt 1948 noch die Hälfte der Bevölkerung den Nationalsozialismus für eine "gute Idee“, die nur „schlecht ausgeführt“ worden sei ${ }^{59}$.

56 Zum vollständigen Text der Rede vgl. Theodor Heuss, Die großen Reden, Tübingen 1965, S. 99-107.

57 Baumgärtner, Reden nach Hitler, S. 342, 340.

58 Zit. nach: Konzentrationslager Bergen-Belsen, S. 253.

59 Fröhlich/Kohlstruck (Hg.), Engagierte Demokraten, S. 11. 